\title{
Ovarian Hemorrhage, CTCAE
}

National Cancer Institute

\section{Source}

National Cancer Institute. Ovarian Hemorrhage, CT CAE. NCI Thesaurus. Code C56553.

A disorder characterized by bleeding from the ovary. 